\title{
Phase-sensitive high-speed THz imaging
}

\author{
Toshiaki Hattori, Keisuke Ohta, Rakchanok Rungsawang and \\ Keiji Tukamoto \\ Institute of Applied Physics, University of Tsukuba, 1-1-1 Tennodai, Tsukuba, \\ Ibaraki, 305-8573 Japan \\ E-mail: hattori@bk.tsukuba.ac.jp
}

\begin{abstract}
Phase-sensitive high-speed imaging of terahertz radiation was achieved by introducing the optical heterodyne detection method to the high-speed electro-optic sampling of terahertz images. Intense terahertz pulses obtained from a large-aperture photoconductive antenna were used as the source. The electric field distribution on the focal plane of focused half-cycle terahertz pulses was observed using the apparatus. Annular spatial profiles were observed in the time-dependent images. Frequencydependent distribution calculated by means of Fourier transformation showed almost diffraction-limited focusing.
\end{abstract}

PACS numbers: 42.30.Va, 42.65.Re

Submitted to: J. Phys. D: Appl. Phys.

\section{Introduction}

Imaging with terahertz $(\mathrm{THz})$ radiation has been attracting attention because the radiation in this frequency region has only recently become avaliable for imaging, thus allowing phase-sensitive detection to be possible in principle [1]. It takes a long time to obtain images using relatively weak $\mathrm{THz}$ radiation since spatial scanning of the $\mathrm{THz}$ beam is required, which can prohibit applications in the real world. Simultaneous detection of two-dimensional data using a charge coupled device (CCD) camera has made possible high-speed $\mathrm{THz}$ imaging $[2,3]$, where no spatial scanning is needed. We have previously reported a fast $\mathrm{THz}$ imaging method using high-power $\mathrm{THz}$ pulses generated from large-aperture photoconductive antennas [3], where electro-optic (EO) sampling measurements of the two-dimensional spatial distribution of $\mathrm{THz}$ intensity were achieved using a large-area EO crystal, an expanded probe beam, and a CCD camera [3]. In this method, however, the polarizer and the polarization analyzer were oriented perpendicularly to each other, and the spatial distribution of the intensity, or the squared electric field, was obtained. The most commonly adopted ballanced detection method used in EO sampling [4, 5], which directly measures the electric field, is virtually impossible in image detection using a CCD camera. 
The field-linear detection method of nonliear optical signals using the polarization change of the probe light was first developed by Eesley and Levenson [6, 7] for measurements of the Raman-induced Kerr effect. They introduced the optical heterodyne detection (OHD) method, where the optical field generated by the nonlinear optical processes is heterodyne detected by a local oscillator, which is a phase-shifted fraction of the probe light. The OHD technique has since been applied to several schemes aimed at detecting optical Kerr effects [8, 9, 10]. Jiang et al. has first introduced this technique to $\mathrm{THz}$ field detection using the EO sampling method, and derived the optimal bias point under the existence of background light [11].

In this report, we applied for the first time the OHD method for the high-speed imaging of the two-dimensional (2-D) spatial distribution of a $\mathrm{THz}$ field using a CCD camera. A time-dependent spatial distribution of the electric field of focused half-cycle $\mathrm{THz}$ pulses was obtained by the measurements. Annular spatial profiles of the field at time regions slightly apart from the peak were verified, which were first observed using the $\mathrm{THz}$ intensity imaging setup [3]. Fourier analysis of the temporal profile of the field at each position showed the frequency-resolved 2-D spatial profile of the field, which is not possible using the intensity images.

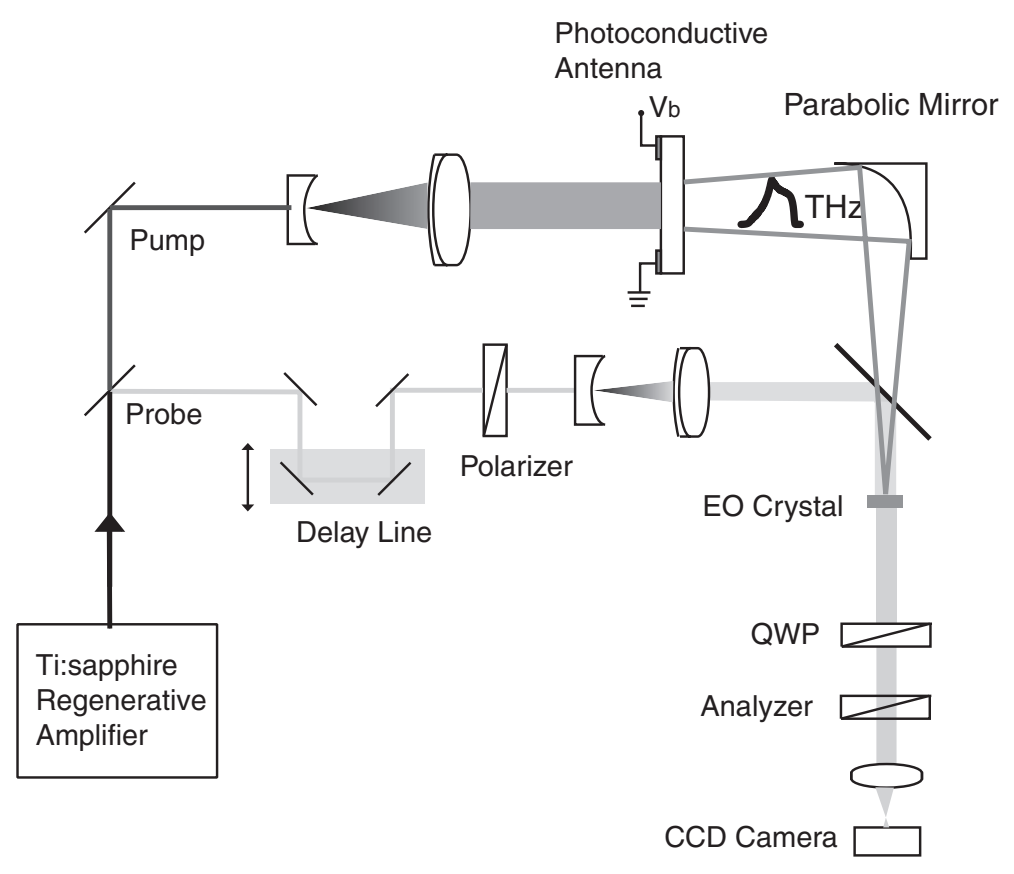

Figure 1. Schematic of the experimental setup. QWP: a quarter-wave plate.

\section{Experimental setup}

The experimental setup is shown in figure 1, which was based on a previously described setup $[3,12,13,14]$. A regeneratively amplified Ti:sapphire laser provides $800 \mathrm{~nm}$ pulses with a duration of $150 \mathrm{fs}$ at a repetition rate of $1 \mathrm{kHz}$. The major part of the output 
was used to pump a large-aperture GaAs photoconductive antenna with a $3 \mathrm{~cm}$ gap between the electrodes. The $\mathrm{THz}$ radiation emitted in the transmitted direction was focused by an off-axis parabolic mirror with a focal length of $152.4 \mathrm{~mm}$. By this setup, almost half-cycle pulses with a subpicosecond duration are obtained [3, 14]. The 2-D spatial distribution of the $\mathrm{THz}$ field on the focal plane was measured by means of $\mathrm{EO}$ sampling with a 1-mm-thick $\langle 110\rangle \mathrm{ZnTe}$ crystal. The ZnTe crystal had an active area of $18 \mathrm{~mm}$ (horizontal) $\times 20 \mathrm{~mm}$ (vertical). A $1 / 5$ image of the spatial profile of the $\mathrm{THz}$ radiation on the crystal position was obtained by the CCD camera. A quarter-wave plate was inserted for electric field detection as described in the following.

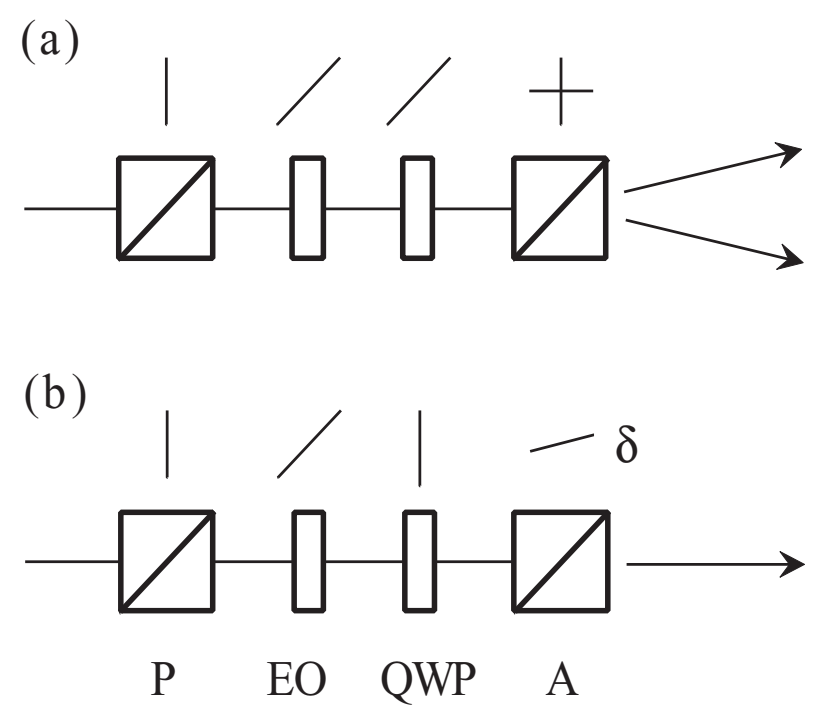

Figure 2. The experimental configuration of the optics in (a) balanced detection, and (b) the OHD method. P: a polarizer, EO: an EO crystal, QWP: a quarter-wave plate, and A: a polarization analyzer. The direction of the optical axis of each component is denoted above it. Vertical lines and $45^{\circ}$ lines show, respectively, that the optical axes of the components are in the vertical and horizontal directions, or in the $45^{\circ}$ direction.

The configuration of the polarizer, EO crystal, wave plate, and polarization analyzer in the OHD method is compared with that of the ballanced detection method shown in figure 2. In the OHD setup, the quarter-wave plate has an axis parallel to the incident light polarization. The analyzer is rotated by a small angle, $\delta$, from the crossed orientation. The intensity of the probe light transmitted through this setup can be expressed as [11]

$$
I=I_{0}\left[\eta+\sin ^{2}(\delta+\theta)\right]
$$

which leads to

$$
\theta=-\delta+\sqrt{\delta^{2}+\frac{I-I_{b}}{I_{0}}}
$$

when $|\delta|,|\theta| \ll 1$. Here, $I_{0}$ is the incident light intensity, $\theta$ is the EO effect contribution to the phase difference, which is proportional to the field, $\eta$ describes the background 
contribution due to the intrinsic birefringence of optics and scattering, and $I_{b}$ is the background intensity;

$$
I_{b}=I_{0}\left(\eta+\delta^{2}\right) .
$$

The modulation depth is optimized by setting the analyzer angle at $\delta \approx \sqrt{\eta}[11]$. In our setup, the background contribution from the optical elements between the polarizer and the analyzer could be described by $\eta=1.0 \times 10^{-3}$, which is the extinction ratio of the setup. Active compensation for the intrinsic birefringence of optical elements was not necessary.

Wu et al. [2] reported field-linear $\mathrm{THz}$ wave detection without the use of a quarterwave plate. In that case, the local oscillator originates from the polarization rotation of the probe light due to small birefringence in the ZnTe crystal, and thus the amplitude of the local oscillator cannot be optimized in a controlled manner and is not spatially uniform. By the use of the quarter-wave plate, a spatially uniform local oscillator can be introduced in a controlled fashion.

Following Jiang et al. [11], the modulation depth, $\gamma$, of the measurement is introduced as

$$
\gamma \equiv \frac{I_{\theta \neq 0}-I_{\theta=0}}{I_{\theta \neq 0}+I_{\theta=0}}
$$

The experimentally obtained $\delta$ dependence of the signal modulation depth is plotted in figure 3 , which shows close agreement with the theoretical one [11]. In the measurements described below, the orientation of the analyzer was set at $\delta=0.02 \mathrm{rad}$, where the modulation depth is almost optimized. To obtain a single image, 30 laser shots were accumulated. Thus the duration required for taking a single image was as short as 30 ms. The experimentally obtained images were converted to the field images after the measurements using equation (2).

In order to confirm the validity of the present measurement method, we compared the temporal waveform of the $\mathrm{THz}$ radiation at the center of the imaged area, which was reconstructed from a time-scanned sequence of images, with that obtained using the conventional balanced detected EO sampling method at the same position [5]. The result is shown in figure 4, showing that the results obtained by the two methods agree very closely with each other. The waveforms show ringings in the tail due to the free induction decay of water vapor molecules. Since the electric field in this part of the waveform has a small negative value, the correct reconstruction of the waveform is not possible using the intensity imaging method, and is possible only by means of the field-linear detection method.

\section{Results}

The time-resolved spatial profiles of the focused electric field of half-cycle pulses on the focal plane obtained with the setup described above are shown in figure 5 . The images correspond to an area of $18.2 \mathrm{~mm} \times 18.2 \mathrm{~mm}$ at the position of the EO crystal. At the 


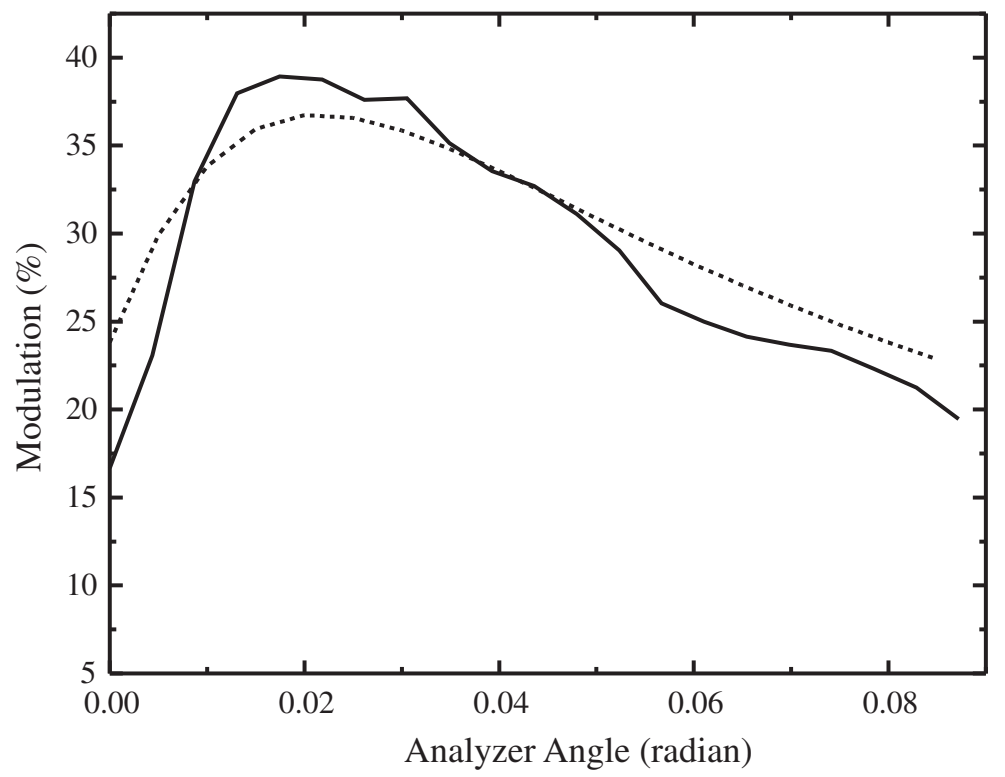

Figure 3. Modulation depth as a function of analyzer angle $\delta$. Solid line shows the experimental result and the dashed line is the theoretical one with $\eta=10^{-3}$ and $\theta=0.025$.

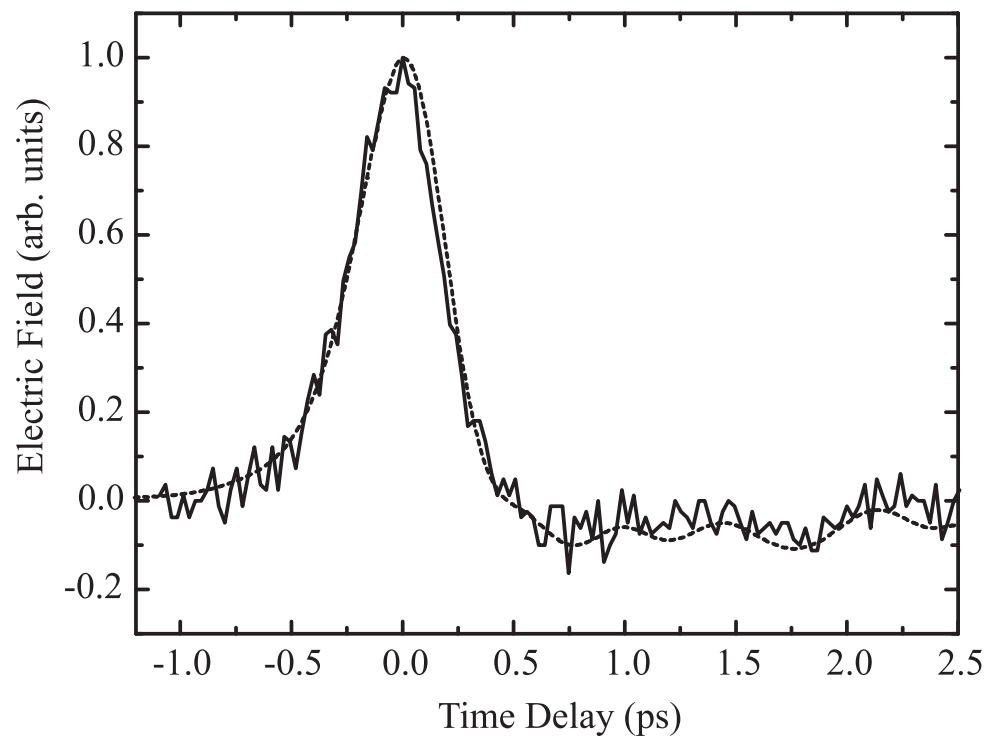

Figure 4. THz temporal waveform obtained using the present OHD imaging method (solid line) and that obtained using conventional balanced detection (dashed line). 
peak time, which is defined as 0 ps, the profile shows a simple Gaussian-like peak at the center, as shown in figure 5(c). On the other hand, there are time regions before and after the peak, where ring-like profiles are observed, as shown in figures $5(\mathrm{a})$ and (d). This feature has been previously observed by $\mathrm{THz}$ intensity image measurements [3], and verified by the field images in the present study. Jiang and Zhang have reported similar behaviors of few-cycle $\mathrm{THz}$ pulses [15], where multiple-ring structures were observed.
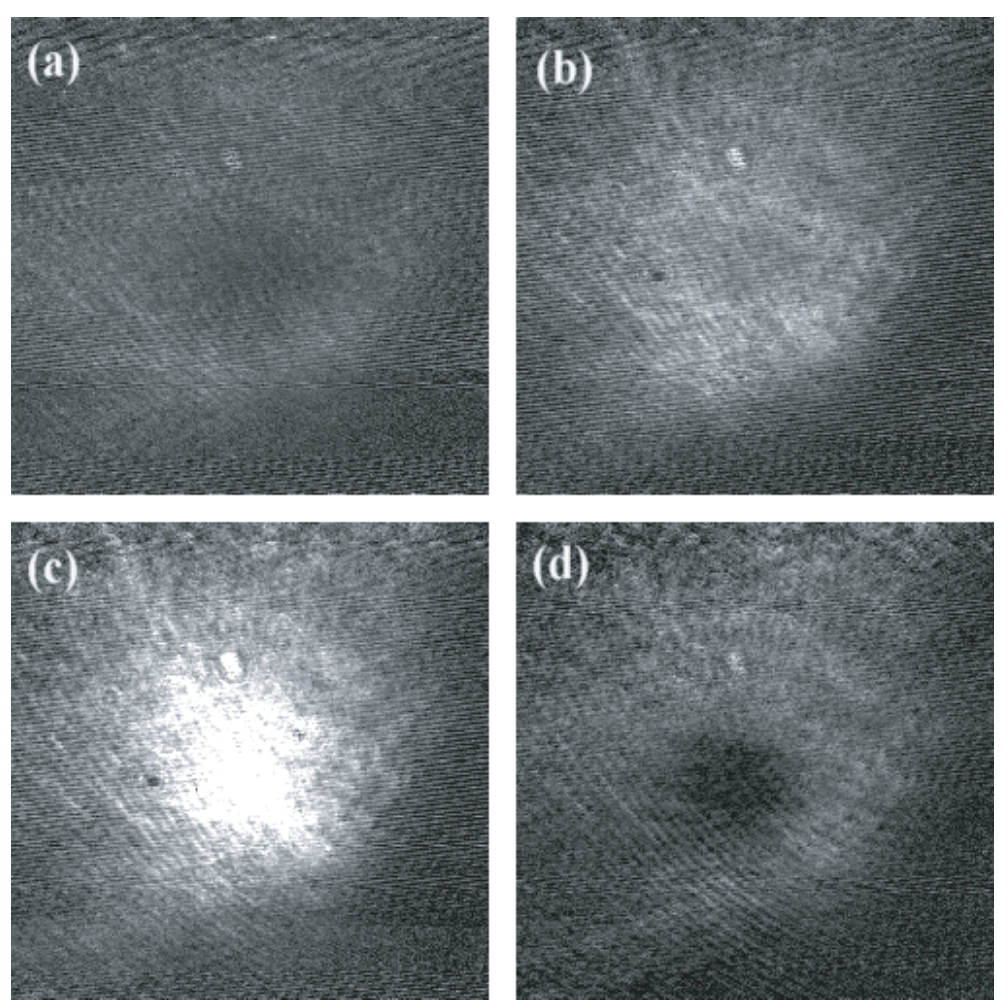

Figure 5. Time-dependence of the 2-D spatial profile of the electric field of a focused half-cycle pulse on the focal plane at (a) -0.8 ps, (b) -0.45 ps, (c) 0 ps (peak time) and (d) 0.45 ps.

The temporal waveform at each position in the image was reconstructed from a time-scanned image sequence consisting of more than one hundred images. By Fourier transforming the field waveform at each position, frequency-resolved spatial distributions of the electric field amplitude were calculated, as shown in figure 6. For better data quality, $4 \times 4$ pixels were combined and the temporal waveforms of the averaged signal intensity were used for the Fourier analysis. The present Fourier analysis is possible only using the field-linear measurements. Using the conventional detection scheme employing crossed polarizers, the squared field is detected. The Fourier transform of the temporal waveform, in that case, corresponds to the self-convolution of the spectrum, which should have much more complicated structures compared with the amplitude spectrum itself. It is clearly seen from the frequency dependence of the amplitude distribution, as shown in the figure, that lower-frequency components are focused to a larger area, and higher-frequency ones to a smaller area. 

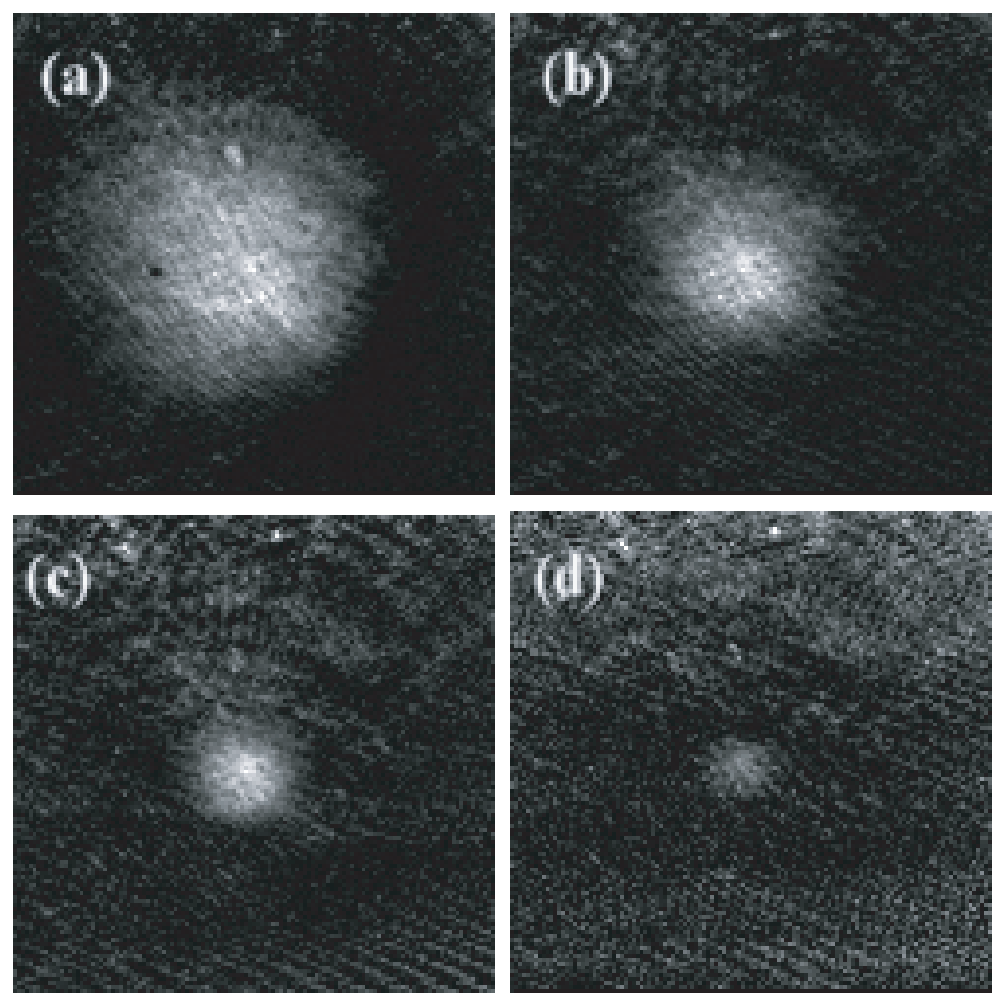

Figure 6. The spatial distribution of the electric field amplitide of (a) $0.48 \mathrm{THz}$, (b) $0.99 \mathrm{THz}$, (c) $1.50 \mathrm{THz}$ and (d) $2.16 \mathrm{THz}$ components of the focused THz pulse. The images correspond to an area of $18.2 \mathrm{~mm} \times 18.2 \mathrm{~mm}$ at the position of the EO crystal. The amplitude is normalized to the peak value at each frequency. The peak value of image (d) is much smaller than that of (a), which enhances the relative level of the background noise of image (d).

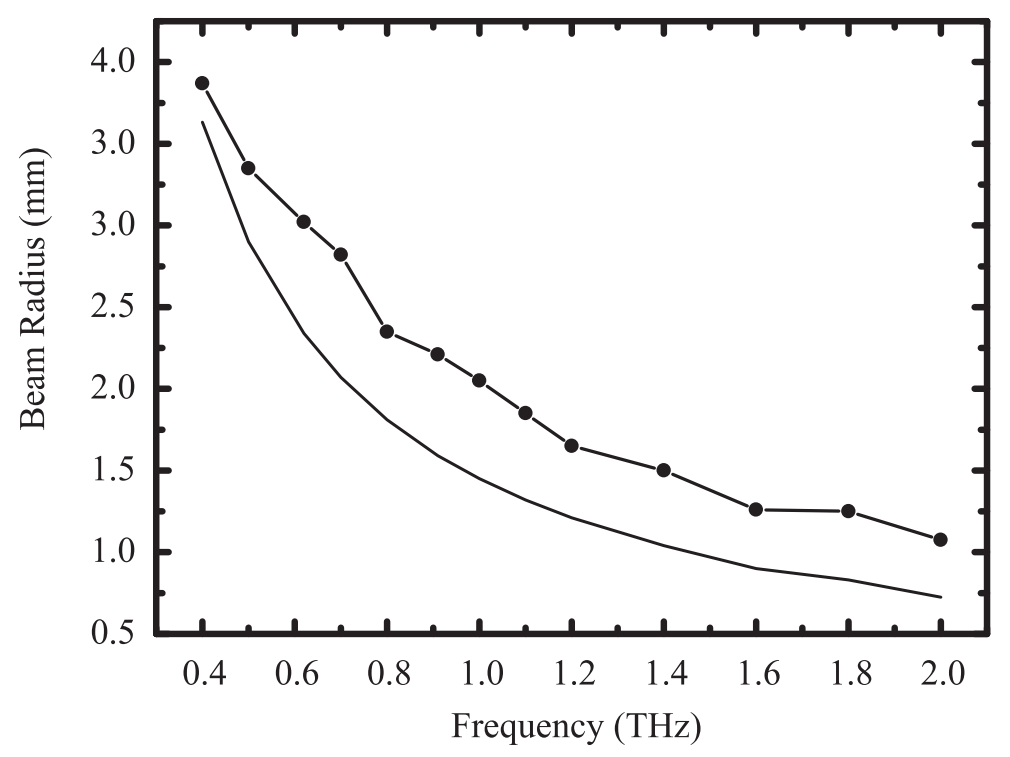

Figure 7. The $\mathrm{THz}$ beam sizes obtained from the frequency-dependent amplitude distribution (solid circles and line) in comparison with the beam-waist sizes based on the Gaussian beam model (line). 
For a more quantitative analysis, the beam size of each frequency component was estimated as follows. First, the amplitude profile on the horizontal line which passes through the center of the image was obtained from each image. Then the $1 / e$ radius of the profile was compared with the analytical expression [13]:

$$
w_{0}=\frac{c f}{A \pi \nu}
$$

which shows the beam-waist size of a focused Gaussian beam of frequency $\nu$ in the highfrequency approximation. Here, $c$ is the speed of light, $f$ is the focal length, and $A=10.0$ $\mathrm{mm}$ is the beam size of the colimated beam before focusing. This expression shows the diffraction limit for each frequency component. A comparison is shown in figure 7, which exhibits close agreement between the experimental and the Gaussian beam results. Several reports have shown that the essential features of spatio-temporal behaviors of $\mathrm{THz}$ pulses can be explained using the Gaussian beam model $[3,13,16]$. In the present beam size comparison, the agreement is very close at low frequencies, which shows that the focusing is almost diffraction limited. At high frequencies, a deviation as high as $50 \%$ of the analytical value is seen, which can be partly attributed to misalignment of optical elements, or the limited spatial resolution of the measurement. Although the measurements of the spatial distribution of $\mathrm{THz}$ energy or amplitude by spatially scanning a small aperture can suffer from the cutoff effects of the aperture $[14,17]$, the present method using free-space EO sampling is not affected by such effects.

\section{Conclusion}

High-speed phase-sensitive time-resolved electric field image detection was achieved by applying the optical heterodyne detection technique for $\mathrm{THz}$ imaging measurements using a CCD camera. The introduction of the local oscillator for heterodyne detection was controlled using a quarter-wave plate. By measurement of the time-resolved 2-D field distribution of a focused half-cycle $\mathrm{THz}$ pulse, a ring-like field distribution in the time-resolved images and almost diffraction-limited focusing in the frequency-resolved images were observed.

\section{Acknowledgments}

This study was partly supported by KAKENHI (13450026) from the Japan Society for the Promotion of Science.

\section{References}

[1] Mittleman D M, Gupta M, Neelamani R, Baraniuk R G, Rudd J V and Koch M 1999 Appl. Phys. B 681085

[2] Wu Q, Hewitt T D and Zhang X-C 1996 Appl. Phys. Lett. 691026

[3] Rungsawang R, Ohta K, Tukamoto K and Hattori T 2003 J. Phys. D 36229

[4] Valdmanis J A, Mourou G and Gabel C W 1982 Appl. Phys. Lett. 41211 
[5] Nahata A, Weling A S and Heinz T F 1996 Appl. Phys. Lett. 692321

[6] Eesley G L, Levenson M D and Tolles W M 1978 J. Quantum Electron. 1445

[7] Levenson M D and Eesley G L 1979 Appl. Phys. 191

[8] Ippen E P and Shank C V 1975 Appl. Phys. Lett. 2692

[9] McMorrow D, Lotshaw W T and Kenney-Wallace G A 1988 J. Quantum Electron. 24443

[10] Hattori T, Terasaki A, Kobayashi T, Wada T, Yamada A and Sasabe H 1991 J. Chem. Phys. 95 937

[11] Jiang Z, Sun F G, Chen Q and Zhang X-C 1999 Appl. Phys. Lett. 741191

[12] Hattori T, Tukamoto K and Nakatsuka H 2001 Jpn. J. Appl. Phys. 404907

[13] Hattori T, Rungsawang R, Ohta K and Tukamoto K 2002 Jpn. J. Appl. Phys. 415198

[14] Tukamoto K, Rungsawang R and Hattori T 2003 Jpn. J. Appl. Phys. 421609

[15] Jiang Z and Zhang X-C 1999 Opt. Express 5243

[16] You D and Buchsbaum P H 1997 J. Opt. Soc. Am B 141651

[17] Bromage J, Radic S, Agrawal G P and Stroud Jr. C R 1998 J. Opt. Soc. Am B 151953 